\title{
The Positive Action Conjecture and Asymptotically Euclidean Metrics in Quantum Gravity
}

\author{
G. W. Gibbons and C. N. Pope \\ Department of Applied Mathematics and Theoretical Physics, University of Cambridge, \\ Cambridge CB3 9EW, England
}

\begin{abstract}
The Positive Action conjecture requires that the action of any asymptotically Euclidean 4-dimensional Riemannian metric be positive, vanishing if and only if the space is flat. Because any Ricci flat, asymptotically Euclidean metric has zero action and is local extremum of the action which is a local minimum at flat space, the conjecture requires that there are no Ricci flat asymptotically Euclidean metrics other than flat space, which would establish that flat space is the only local minimum. We prove this for metrics on $R^{4}$ and a large class of more complicated topologies and for self-dual metrics. We show that if $R_{\mu}^{\mu} \geqq 0$ there are no bound states of the Dirac equation and discuss the relevance to possible baryon non-conserving processes mediated by gravitational instantons. We conclude that these are forbidden in the lowest stationary phase approximation. We give a detailed discussion of instantons invariant under an $S U(2)$ or $S O(3)$ isometry group. We find all regular solutions, none of which is asymptotically Euclidean and all of which possess a further Killing vector. In an appendix we construct an approximate self-dual metric on $\mathrm{K} 3$ - the only simply connected compact manifold which admits a self-dual metric.
\end{abstract}

\section{Introduction}

It has been expected for some time $[1,2,3]$ that matter should be unstable when quantum gravity is taken into account. That is one expects gravity at some nonperturbative level to give rise to baryon and lepton number non-conservation. This is most clearly indicated in the external field theory computations of black hole evaporation [2]. One would like to compute processes of this sort using a fully quantized theory of gravity. The version of Quantum Gravity which seems most appropriate to us is the functional approach.

In the functional integral formulation of flat space quantum field theory physical quantities are expressed formally as functional integrals of the form

$$
Z=\int_{C} d[\varphi] O[\varphi] \operatorname{expiI}[\varphi] .
$$


$d[\varphi]$ is some measure on the space of field configurations. $I[\varphi]$ is the classical action functional and $O[\varphi]$ is some classical functional of the field $\varphi$ whose quantum mechanical expectation one wishes to calculate. $C$ denotes the class of field configurations that enter the sum and is specified by giving suitable boundary conditions for $\varphi$. The freedom to choose $C$ corresponds to choosing the states that enter the matrix element. As a preliminary step to defining (1.1) one "Wick rotates" to Euclidean 4-space, $\left\{\mathbb{R}^{4}, \delta_{\alpha \beta}\right\}$ thus making the argument of the exponential real and negative. All fields are then supposed to die away at large spatial distances. The vacuum persistence amplitude corresponds to fields which in addition die away at large positive or negative imaginary times $\tau$. If instead one wishes to compute the grand canonical partition function for a boson system at some temperature $T$ one includes in the sum only those fields which are periodic in imaginary time with period $\beta=T^{-1}$. This case is equivalent to working on a flat space with the time coordinate identified - i.e. on $\left\{\mathbb{R}^{3} \times S^{1}, \delta_{\alpha \beta}\right\}$. In both cases one can regard the manifold as the limit of a compact manifold with a boundary which is moved to infinity.

To evaluate the functional integral one first looks for non-singular stationary points of the action functional (classical solutions) and expands about them. Such critical points are called "Instantons".

In Quantum Gravity one might try to imitate this procedure by summing over all 4-dimensional Riemannian spaces $\left\{M, g_{\alpha \beta}\right\}$ with arbitrary topology for the manifold $M$ and arbitrary metric $g_{\alpha \beta}$ except that $M$ has a prescribed boundary $\partial M$ and $g_{\alpha \beta}$ induces on $\partial M$ some prescribed geometry with metric $k_{\alpha \beta}$. At finite temperature the relevant instanton is the Schwarzschild solution [4]. Presumably the appropriate boundary condition for the vacuum persistence amplitude corresponds to metrics which are asymptotically Euclidean and whose boundary at infinity can be regarded as a 3-sphere with its standard metric. That is, an asymptotically Euclidean metric is one such that outside a compact set the manifold is diffeomorphic to $R^{4}$ with a closed ball removed and the metric tends to the standard flat Euclidean metric at least as fast as $r^{-2}$, where $r$ is the asymptotic proper radial distance. The condition that the metric tend to flatness as $r^{-2}$ guarantees that the action is finite.

In [5] it was argued that to evaluate the functional integral one should pick in each conformal equivalence class a metric satisfying $R_{\mu}^{\mu}=0$. One can then explicitly integrate over conformal deformations of that metric. One is then left with the task of summing over all metrics satisfying $R_{\mu}^{\mu}=0$. The action of these metrics is given by a boundary term (the volume term vanishes). The path integral would be better behaved if the action were positive, vanishing if and only if the metric were flat. This is called the "Positive Action Conjecture" $[5,6]$. It is rather plausible since it is the natural generalization to one higher dimension of the familiar Positive Mass Conjecture - a proof of which has recently been announced [7]. If the Positive Action Conjecture is true there can be no non-trivial asymptotically Euclidean solution of the Einstein equations. This is because the action of such a solution would be zero, which may be seen as follows. Under a constant rescaling of the metric : $g_{\alpha \beta} \rightarrow \Omega^{2} g_{\alpha \beta}, \Omega$ a constant, $I \rightarrow \Omega^{2} I$. For a solution $I$ must be stationary and hence must vanish. Furthermore since flat space is a local minimum of the action the non-existence of non-flat Ricci-flat asymptotically Euclidean metrics would 
establish that the action has a unique local minimum at flat space which is strong evidence (but of course not conclusive proof) that it is always positive. Thus the non-existence of non-flat asymptotically Euclidean solutions is necessary and almost sufficient for the validity of the full Positive Action Conjecture.

This situation is in marked contrast with that in Yang-Mills theory where there exist finite action topologically non-trivial classical solutions which it is believed can be responsible for tunnelling between topologically inequivalent vacua $\left|O_{Y M}, n\right\rangle$ labelled by an integer $n$ [8]. These classical solutions (instantons) have self-dual field strengths. This implies the existence of bound states of the Dirac equation. Because of these bound states the amplitude for a transition between the state $\left|O_{Y M}, n\right\rangle$ and $\left|O_{Y M}, n+1\right\rangle$ whilst the Dirac field remains in the no particle state $\left|O_{D}\right\rangle$ is zero [8].

't Hooft has interpreted this as meaning that tunnelling between topologically inequivalent vacua must be accompanied by a change in chirality of the fermions. In fact he finds that the amplitude

$$
\left\langle O_{D}\left|\otimes\left\langle O_{Y M}, n \mid O_{D}\right\rangle \otimes\right| O_{Y M}, n+1\right\rangle
$$

is non-zero only if a suitable external source is provided to alter the chirality. This can in turn lead to baryon and lepton non-conservation.

For gravity things are different. Firstly because of the scaling behaviour of the gravitational action there is no barrier to topology change [9]. Secondly for noncompact metrics with $R_{\mu}^{\mu} \geqq 0$ there will be no bound states of the Dirac equation. This is because such solutions must be covariantly constant and hence not normalizable [10]. Thirdly because of the Positive Action Conjecture we expect no asymptotically Euclidean solutions. Certainly there are no self-dual solutions as will be shown in Sect. 3. One way out of this situation is to consider spin $\frac{3}{2}$ fields on non-asymptotically Euclidean self-dual metrics [11].

A different way in which baryon non-conservation might arise is via metrics with more than one asymptotically Euclidean region. These are the 4-dimensional analogues of the 3-dimensional "wormholes" and Einstein-Rosen throats of black hole physics $[12,13,14,15,16]$. Indeed if the Ricci scalar, $R_{\mu}^{\mu}$, vanishes they provide initial data for time-symmetric spacetimes in 5-dimensional relativity. The asymptotically Euclidean regions are connected by minimal 3-surfaces which are the intersection of the initial 4-surface with the 4-dimensional apparent horizons in the 5-dimensional Lorentzian manifold which we call, following traditional literary usage, Hyperspace. One may generalize the usual Cosmic Censorship Hypothesis [15, 16, 17, 18] to Hyperspace. If it is true it requires that an asymptotically Euclidean region containing an outermost minimal 3-surface with 3 volume $V_{3}$ should have an action $I$ satisfying

$$
I \geqq\left(\frac{27}{2^{14} \pi}\right)^{1 / 3} V_{3}^{2 / 3} .
$$

Thus these wormholes should be far from stationary points and should require large excursions in the action. They would therefore be damped in the functional integral. Nevertheless they may be responsible for baryon and lepton nonconservation. This might occur if for instance a baryon fell in imaginary time from one asymptotic region to another through a hole while a lepton travelled in the 
opposite direction to replace it [9]. The fact that such configurations are far from stationary points is presumably related to the observed great stability of matter.

In this paper we shall investigate asymptotically Euclidean classical solutions with $N$ asymptotic regions and show that if any do exist their Euler number $\chi$ and signature $\tau$ must satisfy

$$
2 \chi-3|\tau|>2 N \text {. }
$$

This inequality is sufficient for example to rule out asymptotically Euclidean solutions on $R^{4}$ with $N-1$ points removed or on $S^{1} \times S^{3}$ with $N$ points removed. Thus manifolds with wormholes and bridges cannot be solutions.

We also give as an illustration of these ideas a detailed discussion of 4-metrics invariant under the action of $S O(3)$ or $S U(2)$ acting on 3 surfaces (i.e. Bianchi IX metrics). The equations for self-dual metrics can be reduced to first order ordinary differential equations for which we find all non-singular solutions. We give a qualitative discussion of the non-self-dual case and argue that the only nonsingular solutions must admit a further Killing vector and we find all solutions in this class, relating them to various special cases in the literature.

The paper is in 6 sections. Section 2 contains definitions and examples of asymptotically Euclidean metrics. In 3 we prove our main theorems and apply them. In 4 we set up the Bianchi IX formalism and derive the equations for selfdual solutions. In 5 we discuss the boundary conditions and relate them to the nuts and bolts classification of gravitational instanton symmetries [19] and in Sect. 6 we give the qualitative discussion of the solutions. As an application of the solutions we construct in an appendix an approximate metric on $K 3$ - the only simple connected compact manifold to admit a self-dual metric [20].

\section{Examples of Asymptotically Euclidean Metrics}

We define any asymptotically Euclidean region to be one admitting a chart $\left\{x^{\mu}\right\}$ such that for $\left(x_{\mu} x^{\mu}\right)^{1 / 2}=r>r_{0}$ the metric can be written as

$$
g_{\mu \nu}=\left(1+\frac{a^{2}}{4 r^{2}}\right)^{2} \delta_{\mu \nu}+h_{\mu \nu}
$$

where

$$
\begin{aligned}
h_{\mu \nu} & =O\left(\frac{1}{r^{3}}\right) \\
\partial_{r}^{p} \partial_{T}^{q} h_{\mu \nu} & =O\left(\frac{1}{r^{3+p}}\right)
\end{aligned}
$$

and $\partial_{r}$ and $\partial_{T}$ denote radial and transverse derivatives respectively.

The Gravitational Action is

$$
I=-\frac{1}{16 \pi} \int_{M} R \sqrt{g} d^{4} x-\frac{1}{8 \pi} \int_{\partial M}[K] \sqrt{k} d^{3} x
$$

where

$$
k_{\mu \nu}=g_{\mu \nu}-n_{\mu} n_{v}
$$


is the metric induced on the boundary $\partial M$ whose unit normal is $n_{\mu}$. $[K]=K-K_{0}$ is the difference between the trace of the second fundamental form of the boundary $\partial M$ in the metric $g_{\mu \nu}$ and its value in the flat metric $\delta_{\mu \nu}$. In fact

$$
\int_{\partial M} K \sqrt{k} d^{3} x=\frac{\partial V_{3}}{\partial \xi}
$$

where $V_{3}$ is the 3-volume of $\partial M$ and $\frac{\partial}{\partial \xi}$ denotes derivation with respect to the proper distance along the outward normal to $\partial M$. For flat space $V_{3}=2 \pi^{2} \xi^{3}$. Therefore we take $\int_{\partial M} K_{0} \sqrt{k} d^{3} x$ to be $6 \pi^{2}\left(\frac{V_{3}}{2 \pi^{2}}\right)^{2 / 3}$.

For metrices of the form (2.1)

$$
R_{\mu \nu}=O\left(\frac{1}{r^{4}}\right)
$$

but

$$
R_{\mu}^{\mu}=O\left(\frac{1}{r^{5}}\right)
$$

The volume term in (2.4) will therefore converge. The boundary term in (2.4) for a surface of the form $r=R$ is

$$
\frac{3 \pi a^{2}}{8}+O\left(\frac{1}{R}\right)
$$

This evidently converges as $R \rightarrow \infty$. The expression

$$
I=-\frac{1}{16 \pi} \int_{M} R \sqrt{g} d^{4} x-\frac{1}{8 \pi} \int_{\partial M} K \sqrt{k} d^{3} x+6 \pi^{2}\left(\frac{1}{2 \pi^{2}} \int_{\partial M} \sqrt{k} d^{3} x\right)^{2 / 3}
$$

is manifestly coordinate independent but its limiting value could in principle depend on the limiting sequence of boundaries chosen. If the surface is sufficiently spherical this will not happen. The shear, $\Sigma_{\mu \nu}$, of the surface $\partial M$ is defined by

$$
\Sigma_{\mu \nu}=n_{\alpha ; \beta} k_{\mu}^{\alpha} k_{v}^{\beta}-\frac{1}{3} k_{\mu \nu} K
$$

If

$$
\Sigma_{\mu \nu}=O\left(\frac{1}{r^{2}}\right)
$$

then the action will be independent of the limiting sequence.

An example of an asymptotically flat metric with $R_{\mu}^{\mu}=0$ is the Tolman wormhole:

$$
d s^{2}=d \xi^{2}+\left(a^{2}+\xi^{2}\right) d \Omega_{3}^{2} .
$$

This may be obtained as an analytic continuation of the Friedmann-RobertsonWalker cosmological model filled with a perfect fluid whose pressure is $\frac{1}{3}$ its energy density [21]. It contains two asymptotically Euclidean regions, located at large 
positive and negative values of $\xi$, connected by a minimal 3 -sphere at $\xi=0$ whose volume is $2 \pi^{2} a^{3}$. The metric is therefore defined on a manifold of topology $S^{3} \times R \approx R^{4}-\{0\}$ with zero Euler number $\chi$ and signature $\tau$. The action evaluated at each infinity is

$$
I=\frac{3 \pi}{8} a^{2} .
$$

The metric is conformally flat which is made manifest by introducing a radial coordinate $r$ by

$$
\xi=r-\frac{a^{2}}{4 r}
$$

This takes the metric (2.12) to

$$
d s^{2}=\left(1+\frac{a^{2}}{4 r^{2}}\right)^{2}\left(d r^{2}+r^{2} d \Omega_{3}^{2}\right) \text {. }
$$

The asymptotically Euclidean regions are now given by $\infty>r>\frac{a}{2}$ and $\frac{a}{2}>r>0$. The minimal 3-surface is located at $r=\frac{a}{2}$ and the singularity at $r=0$ corresponds to the infinitely distant boundary. The metric is the 4-dimensional analogue of the Einstein-Rosen throat of black hole physics. It may be regarded as a constant time slice of a 5-dimensional hyperspherically symmetric black hole. Such a black hole with vanishing Ricci tensor has the metric

$$
d s^{2}=-\left(1-\frac{a^{2}}{\varrho^{2}}\right)\left(d x^{5}\right)^{2}+\left(1-\frac{a^{2}}{\varrho^{2}}\right)^{-1} d \varrho^{2}+\varrho^{2} d \Omega_{3}^{2}
$$

where $x^{5}$ is the 5 -dimensional time coordinate and $\varrho$ is related to $r$ by

$$
\varrho=r+\frac{a^{2}}{4 r} \text {. }
$$

This example may be readily generalized to the case of a conformally flat 4-metric with vanishing Ricci scalar and $N$ asymptotically Euclidean regions. The metric is

$$
d s^{2}=\left(1+\sum_{i=0}^{N-1} a_{i}^{2}\left[\left(x^{\mu}-x_{i}^{\mu}\right)\left(x^{\nu}-x_{i}^{\nu}\right) \delta_{\mu \nu}\right]^{-1}\right)^{2} \delta_{\alpha \beta} d x^{\alpha} d x^{\beta}
$$

The $N-1$ points $x^{\mu}=x_{i}^{\mu}$ correspond to $N-1$ asymptotic regions. The metric is well defined on $R^{4}-\left\{x_{i}^{\nu}\right\}$. It has Euler number $\chi=1-N$ and vanishing signature $\tau$.

One may also readily generalize the Misner Wormhole [13]. This has the metric

$$
\begin{aligned}
& d s^{2}=\Omega^{2}\left(d \eta^{2}+d \zeta^{2}+\sin ^{2} \zeta d \Omega_{2}^{2}\right) \\
& \Omega=a \sum_{n=-\infty}^{\infty}\left(\cosh \left(\eta+2 n \mu_{0}\right)-\cos \zeta\right)^{-1}
\end{aligned}
$$


If we let $0 \leqq \zeta \leqq \pi$ and $0 \leqq \eta \leqq \eta_{0}$ we obtain a metric on $S^{1} \times S^{3}$. However the metric is singular at $\eta=0, \zeta=0$ which turns out to be an asymptotically Euclidean infinity. The metric is thus well defined on $S^{3} \times S^{1}-\{0\}$ which has Euler number -1 and signature zero.

\section{The Topology of Asymptotically Euclidean Spaces}

It is convenient to think of an asymptotically Euclidean space $M$ as being topologically equivalent to a compact manifold $M_{c}$ with $N$ points removed. A triangulation of $M_{c}$ will serve as a triangulation of $M$ provided one removes $N$ 4-simplices. Thus the Euler number of an asymptotically Euclidean manifold with $N$ "infinities" is given by

$$
\chi[M]=\chi\left[M_{c}\right]-N .
$$

The signature will be unchanged, i.e.

$$
\tau[M]=\tau\left[M_{c}\right] \text {. }
$$

Both $\chi$ and $\tau$ may be related to integrals over $M$ of curvature invariants. The Gauss-Bonnet Theorem states [22, 23]

$$
\chi[M]=\frac{1}{32 \pi^{2}} \int_{M} \varepsilon_{\alpha \beta \gamma \delta} R^{\alpha \beta} \wedge R^{\gamma \delta}-\frac{1}{32 \pi^{2}} \int_{\partial M} \varepsilon_{\alpha \beta \gamma \delta}\left(2 \vartheta^{\alpha \beta} \wedge R^{\gamma \delta}-\frac{4}{3} \vartheta^{\alpha \beta} \wedge \vartheta_{\varepsilon}^{\gamma} \wedge \vartheta^{\varepsilon \delta}\right) .
$$

$R_{\beta}^{\alpha}$ are the curvature 2 -forms in an orthonormal basis and $\vartheta_{\beta}^{\alpha}$ is the second fundamental form of the boundary $\partial M$. That is if $\omega_{\beta}^{\alpha}$ are the actual connection 1 -forms and $\omega_{0 \beta}^{\alpha}$ are the connection 1 -forms if the metric were locally a product near the boundary

$$
\vartheta_{\beta}^{\alpha}=\omega_{\beta}^{\alpha}-\omega_{0 \beta}^{\alpha} .
$$

The second fundamental form may also be defined by constructing a Gaussian coordinate system about each connected component. If $n^{\alpha}$ is the unit normal then

$$
k_{\alpha \beta}=g_{\alpha \beta}-n_{\alpha} n_{\beta} .
$$

One now defines a symmetric tensor $K_{\alpha \beta}$ by

$$
K_{\alpha \beta}=\underset{n}{\mathscr{L}} k_{\alpha \beta} \text {. }
$$

The boundary term in the action, $K$, is

$$
K=K_{\alpha \beta} k^{\alpha \beta}
$$

where

$k^{\alpha \sigma} k_{\sigma \beta}=\delta_{\beta}^{\alpha}$.

In a basis of 1 -forms $\left(\omega^{0}, \omega^{i}\right)$ such that

$$
\omega^{0}=n_{\alpha} d x^{\alpha}
$$

and

$$
k_{\alpha \beta} d x^{\alpha} d x^{\beta}=\sum_{i=1}^{3}\left(\omega^{i}\right)^{2}
$$


we have

$$
\frac{\partial \omega^{i}}{\partial \xi}=\underset{n}{\mathscr{L}} \omega^{i}=\mathrm{K}_{j}^{i} \omega^{j}
$$

The $\vartheta_{\beta}^{\alpha}$ are given by

$$
\begin{aligned}
& \vartheta_{0}^{i}=\frac{\partial \omega^{i}}{\partial \xi}=K_{j}^{i} \omega^{j} \\
& \vartheta_{k}^{i}=0 .
\end{aligned}
$$

For asymptotically Euclidean metrics the second boundary term in (3.3) vanishes and we have

$$
\begin{aligned}
\chi[M] & =\frac{1}{32 \pi^{2}} \int_{M}\left(C_{\mu \nu \alpha \beta} C^{\mu \nu \alpha \beta}-2 R_{\mu \nu} R^{\mu \nu}+\frac{2}{3} R^{2}\right) \sqrt{g} d^{4} x+\frac{1}{2 \pi^{2}} \int_{\partial M} \operatorname{det}\left(K_{j}^{i}\right) \sqrt{k} \mathrm{~d}^{3} \mathrm{x} \\
& =\frac{1}{32 \pi^{2}} \int_{M}\left(C_{\mu \nu \alpha \beta} C^{\mu \nu \alpha \beta}-2 R_{\mu \nu} R^{\mu \nu}+\frac{2}{3} R^{2}\right) \sqrt{g} d^{4} x+N .
\end{aligned}
$$

If $R_{\mu \nu}=0$,

$$
\chi[M] \geqq N
$$

equality being attained if and only if the metric is flat.

The signature may also be expressed in terms of integrals but one must include a non-local boundary contribution [24]:

$$
\tau[M]=\frac{1}{48 \pi^{2}} \int_{M} R_{\alpha}^{\beta} \wedge R_{\beta}^{\alpha}-\frac{1}{48 \pi^{2}} \int_{\partial M} \vartheta_{\beta}^{\alpha} \wedge R_{\alpha}^{\beta}-\eta(0) .
$$

$\eta(s)$ is the $\eta$-function of a certain differential operator defined over $\partial M . \eta(0)$ vanishes if $\partial M$ has an orientation reversing isometry, which $S^{3}$ does. The other boundary term vanishes for asymptotically Euclidean metrics and we obtain

$$
\tau[M]=\frac{1}{48 \pi^{2}} \int_{M} C_{\alpha \beta \gamma \delta} * C^{\alpha \beta \gamma \delta} \sqrt{g} d^{4} x .
$$

${ }^{*} C_{\alpha \beta \gamma \delta}$ is the dual of the Weyl tensor $C_{\alpha \beta \gamma \delta}$. Using the fact that

$$
C_{\alpha \beta \gamma \delta} C^{\alpha \beta \gamma \delta} \geqq\left|C_{\alpha \beta \gamma \delta}{ }^{*} C^{\alpha \beta \gamma \delta}\right|,
$$

equality being attained if and only if the Weyl tensor is self-dual or anti-self-dual we obtain the following inequality for vacuum Einstein solutions

$$
\begin{aligned}
& 2 \chi[M]-3|\tau[M]| \geqq 2 N \\
& 2 \chi\left[M_{c}\right]-3\left|\tau\left[M_{c}\right]\right| \geqq 4 N .
\end{aligned}
$$

Similar inequalities have previously been obtained by Hitchin in the compact case [20].

One might think that equality could be obtained in the half-flat case. However complete self-dual asymptotically Euclidean metrics (at least with finite fundamen- 
tal group) do not exist. This can be seen as follows. The index of the Dirac operator $\gamma^{\mu} \nabla_{\mu}$ on a manifold with boundary is given by [24]

$$
\text { Index }\left[\gamma^{\alpha} \nabla_{\alpha}\right]=\frac{1}{192 \pi^{2}} \int_{M} R_{\alpha}^{\beta} \wedge R_{\beta}^{\alpha}-\frac{1}{192 \pi^{2}} \int_{\partial M} \vartheta_{\beta}^{\alpha} \wedge R_{\alpha}^{\beta}-\left[\eta_{D}(0)+h\right] \frac{1}{2} .
$$

$\eta_{D}(s)$ is the $\eta$ function of the Dirac operator restricted to the boundary $\partial M$ and $h$ is the dimension of its kernel. For $S^{3}$ these vanish. Now any simply connected halfflat metric posseses spinor structure [25]. Thus on half flat asymptotically Euclidean metric the index of the Dirac operator is

$$
\pm \frac{1}{48 \pi^{2}} \int_{M} R_{\alpha \beta \mu \nu} R^{\alpha \beta \mu \nu} \sqrt{g} d^{4} x .
$$

This is zero only if the metric is flat.

It follows that any simply connected asymptotically Euclidean half flat metric would admit at least one normalizable solution of the Dirac equation. However by Lichnerowicz's Theorem [10] such a solution would have to be covariantly constant and hence could not be normalizable. The only way out of this contradiction is that there are no simply connected half-flat asymptotically Euclidean solutions. If the manifold is not simply connected we can apply the argument to the universal covering space provided this consists of only finitely many copies of the original manifold. Thus the inequalities (3.20) and (3.21) are saturated only in the trivial flat case. Using them it is easy to rule out (amongst others) solutions with the following topologies:

1) $R^{4}-\{N$ points $\} \equiv S^{4}-\{N+1$ points $\}$

$$
\chi=1-N, \quad \tau=0
$$

2) $S^{1} \times S^{3}-\{N$ points $\}$

$$
\chi=-N, \quad \tau=0
$$

3) $C P^{2}-\{N$ points $\}$

$$
\chi=3-N, \quad \tau=1 .
$$

Thus we see that there are no asymptotically Euclidean solutions (other than flat) on $R^{4}$ nor with the topology of the many bridge (2.18) or Misner Wormhole (2.19).

\section{Local Bianchi IX Solutions}

In the next 3 sections we shall illustrate the ideas we have discussed above by reference to a simple class of solutions of the Einstein Equations. This class we call Bianchi IX metrics. They are defined to be metrics with an $S U(2)$ or $S O(3)$ isometry group acting transitively on 3-surfaces. These are the Euclidean equivalent of a well known class of cosmological models [26]. If $R_{\mu \nu}=0$ the metric may locally be cast in the form

$$
d s^{2}=(a b c)^{2} d \eta^{2}+a^{2} \sigma_{1}^{2}+b^{2} \sigma_{2}^{2}+c^{2} \sigma_{3}^{2}
$$


where $a, b$ and $c$ are functions solely of $\eta$ and $\left\{\sigma_{i}\right\}$ are 3 basis 1-forms with exterior algebra

$$
d \sigma_{i}=-\frac{1}{2} \varepsilon_{i j k} \sigma_{j} \wedge \sigma_{k}
$$

and such that

$$
\underset{\partial / \partial n}{\mathscr{L}} \sigma_{i}=0
$$

The vacuum Einstein equations reduce to the form:

$$
2 \alpha_{\eta \eta}=a^{4}-\left(b^{2}-c^{2}\right)^{2}
$$

plus the two equations obtained by cyclic permutation of $(a, b, c)$ and

$$
\begin{aligned}
& 4\left(\alpha_{\eta} \beta_{\eta}+\beta_{\eta} \gamma_{\eta}+\gamma_{\eta} \alpha_{\eta}\right)=2 a^{2} b^{2}+2 b^{2} c^{2}+2 c^{2} a^{2}-a^{4}-b^{4}-c^{4} . \\
& \alpha=\log a
\end{aligned}
$$

and cyclically.

(4.5) is a first integral of (4.4) and may be regarded as a constraint on the initial values of $(a, b, c)$ and $\left(a_{\eta}, b_{\eta}, c_{\eta}\right)$ which is preserved by the evolution Eq. (4.4). In fact (4.4) are derivable as the Euler Lagrange equations of the action $I$ given by

$$
\begin{aligned}
-\frac{8}{\pi} I= & \int 2\left(\alpha_{\eta} \beta_{\eta}+\beta_{\eta} \gamma_{\eta}+\gamma_{\eta} \alpha_{\eta}\right) \alpha \eta \\
& +\frac{1}{2} \int\left(2 a^{2} b^{2}+2 b^{2} c^{2}+2 c^{2} a^{2}-a^{4}-b^{4}-c^{4}\right) d \eta .
\end{aligned}
$$

In fact $I$ coincides with the gravitational action if the 3 -volume of the surfaces of constant $\eta$ is taken to be $16 \pi^{2}(a b c)$. The constraint (4.5) may also be derived from the action (4.7) if one imposes the condition that the integral is stationary under the replacement $d \eta \rightarrow N(\eta) d \eta$ where $N(\eta)$ is an arbitrary function of $\eta$. The constraint says that the "Hamiltonian" corresponding to the Lagrangian (4.7) vanishes. Note that because the time is imaginary the roles of the physical Lagrangian and minus the Hamiltonian are interchanged.

Equations (4.4) and (4.5) may be integrated completely if we impose the condition that two of the invariant directions have equal magnitude. E.g. $a=b$. This leads to the general Taub-NUT family which is invariant under a 4-parameter group with the Lie algebra of $U(2)$ :

$$
\begin{aligned}
& a^{2}=b^{2}=\frac{1}{4} q \sinh q\left(\eta-\eta_{2}\right) \operatorname{cosech}^{2} \frac{1}{2} q\left(\eta-\eta_{1}\right) \\
& c^{2}=q \operatorname{cosech} q\left(\eta-\eta_{2}\right) .
\end{aligned}
$$

$\left(q, \eta_{1}, \eta_{2}\right)$ are constants of integration. The more familiar form

$$
\begin{aligned}
d s^{2}= & \left(r^{2}-n^{2}\right)\left(r^{2}-2 m r+n^{2}\right)^{-1} d r^{2} \\
& +4 n^{2}\left(r^{2}-2 m r+n^{2}\right)\left(r^{2}-n^{2}\right)^{-1} \sigma_{3}^{2}+\left(r^{2}-n^{2}\right)\left(\sigma_{1}^{2}+\sigma_{2}^{2}\right)
\end{aligned}
$$

is obtained using the transformation:

$$
\begin{aligned}
& n^{2}=-\frac{1}{4} q \operatorname{cosech} q\left(\eta_{2}-\eta_{1}\right) \\
& m=n \cosh q\left(\eta_{2}-\eta_{1}\right) \\
& r=\frac{q}{4 n}\left(\operatorname{coth} \frac{1}{2} q\left(\eta-\eta_{1}\right)-\operatorname{coth} q\left(\eta_{2}-\eta_{1}\right)\right) .
\end{aligned}
$$


Two special cases are of note

A) $\eta_{1}=\eta_{2}$.

These are the Eguchi-Hanson metrics [27,28]. Real $q$ corresponds to their type II. Imaginary $q$ corresponds to their type I. One can obtain their metric from (4.10) by the following transformation

$$
\begin{gathered}
m=n+\frac{a^{4}}{128 n^{3}} \\
r=m+\frac{\varrho^{2}}{8 n}
\end{gathered}
$$

where $a$ is Eguchi and Hanson's parameter. One now lets $n \rightarrow \infty$. This gives the metric

$$
\begin{aligned}
& d s^{2}=\left(1-\frac{a^{4}}{\varrho^{4}}\right)^{-1} d \varrho^{2}+\frac{1}{4} \varrho^{2}\left(1-\frac{a^{4}}{\varrho^{4}}\right) \sigma_{3}^{2}+\frac{1}{4} \varrho^{2}\left(\sigma_{1}^{2}+\sigma_{2}^{2}\right) \\
& \text { B) } q=0 .
\end{aligned}
$$

This corresponds to the self-dual solution discussed by Hawking [29].

Both A) and B) have self-dual curvature.

All Bianchi IX solutions with self-dual curvature may be obtained systematically. The self-dual conditions leads, after a single integration to the following equations

$$
2 \frac{d \alpha}{d \eta}=b^{2}+c^{2}-a^{2}-2 \lambda_{1} b c
$$

together with the two others obtained by cyclically permuting $(a, b, c)$ and $\left(\lambda_{1}, \lambda_{2}, \lambda_{3}\right)$. The $\left\{\lambda_{i}\right\}$ are constants obeying

$\lambda_{1}=\lambda_{2} \lambda_{3}$ and cyclically .

The possible solutions of (4.19) are

C) $\left(\lambda_{1}, \lambda_{2}, \lambda_{3}\right)=(0,0,0)$

D) $\left(\lambda_{1}, \lambda_{2}, \lambda_{3}\right)=(1,1,1)$

E) $\left(\lambda_{1}, \lambda_{2}, \lambda_{3}\right)=(-1,-1,+1)$

and cyclic permutations. In fact case $E$ is not distinct from case $\mathrm{D}$ since it may be obtained by the substitution $c \rightarrow-c$. Both $C$ and $D$ yield in (4.18) first integrals of the evolution Eq. (4.4) which are consistent with the constraint (4.5).

Case $\mathrm{C}$ may be obtained directly without integration by requiring that the connection forms in the basis $\left(a b c d \eta, a \sigma_{1}, b \sigma_{2}, c \sigma_{3}\right)$ be self-dual, and the resulting Eq. (4.18) may be solved completely to give a metric of the form [28]

$$
d s^{2}=F^{-1 / 2} d \varrho^{2}+F^{1 / 2} \frac{\varrho^{2}}{4}\left[\left(1-\frac{a_{1}^{4}}{\varrho^{4}}\right)^{-1} \sigma_{1}^{2}+\left(1-\frac{a_{2}^{4}}{\varrho^{4}}\right)^{-1} \sigma_{2}^{2}+\left(1-\frac{a_{3}^{4}}{\varrho^{4}}\right)^{-1} \sigma_{3}^{2}\right]
$$

where

$$
F=\left(1-\frac{a_{1}^{4}}{\varrho^{4}}\right)\left(1-\frac{a_{2}^{4}}{\varrho^{4}}\right)\left(1-\frac{a_{3}^{4}}{\varrho^{4}}\right)
$$

and $\left(a_{1}, a_{2}, a_{3}\right)$ are constants. 
We have been unable to integrate case D explicitly except when $a=b$ which leads to the self-dual Taub-NUT metric obtained from (4.10) by setting $m=n$. We shall give a qualitative treatment of the equation for case D in Sect. 6 .

An alternative way of obtaining self-dual metrics is to require that they be vacuum-Kaehler metrics. This implies and is implied by self-duality of the Riemann tensor [30]. This leads to a non-linear partial differential equation for the Kaehler function $K\left(\zeta^{1}, \zeta^{2}, \bar{\zeta}^{1}, \bar{\zeta}^{2}\right)$ considered as a function of the two complex variables $\zeta^{i}, i=1,2$. This equation is

$$
\operatorname{det}\left(\frac{\partial^{2} K}{\partial \zeta^{i} \partial \bar{\zeta}^{j}}\right)=1 \text {. }
$$

If one assumes that $K$ is a function solely of $R^{2}=\left|\zeta^{1}\right|^{2}+\left|\zeta^{2}\right|^{2}$ (4.25) may integrated to give

$$
K=\varrho^{2}+\frac{1}{2} a^{2} \log \left(\frac{\varrho^{2}-a^{2}}{\varrho^{2}+a^{2}}\right)
$$

where

$$
\varrho^{4}-a^{4}=R^{4} .
$$

This leads directly to the Eguchi-Hanson metric (4.17) where

$$
\begin{aligned}
\frac{1}{4} R^{2}\left(\sigma_{1}^{2}+\sigma_{2}^{2}\right) & =\left|d \zeta^{1}\right|^{2}+\left|d \zeta^{2}\right|^{2}-R^{-2}\left|\bar{\zeta}^{1} d \zeta^{1}+\bar{\zeta}^{2} d \zeta^{2}\right|^{2} \\
R^{2} \sigma_{3}^{2} & =\left(\bar{\zeta}^{1} d \zeta^{1}+\bar{\zeta}^{2} d \zeta^{2}-\zeta^{1} d \bar{\zeta}^{1}-\zeta^{2} d \bar{\zeta}^{2}\right)^{2} .
\end{aligned}
$$

One may easily extend this class of solutions to include the effect of the cosmological constant. In order to be an Einstein-Kaehler metric the Kaehler function must satisfy

$$
\operatorname{det}\left(\frac{\partial^{2} K}{\partial \zeta^{i} \partial \bar{\zeta}^{j}}\right)=e^{-1 / 2 \Lambda K}
$$

The assumption that $K$ depend only on $\left|\zeta^{1}\right|^{2}+\left|\zeta^{2}\right|^{2}=R^{2}$ leads to the metric

$$
d s^{2}=\left(1-\frac{a^{4}}{\varrho^{4}}-\frac{1}{6} \Lambda \varrho^{2}\right)^{-1} d \varrho^{2}+\frac{1}{4} \varrho^{2}\left(1-\frac{a^{4}}{\varrho^{4}}-\frac{1}{6} \Lambda \varrho^{2}\right) \sigma_{3}^{2}+\frac{1}{4} \varrho^{2}\left(\sigma_{1}^{2}+\sigma_{2}^{2}\right)
$$

where $\left\{\sigma_{i}\right\}$ are related to $\left\{\zeta^{i}\right\}$ by Eq. (4.28) and (4.29). $K$ is obtained by solving the ordinary differential equation

$$
\frac{d K}{d R} \frac{d}{d R}\left(R \frac{d K}{d R}\right)=8 R^{2} e^{-1 / 2 \Lambda K}
$$

\section{Global Aspects of Bianchi IX Solutions}

The discussion in Sect. 4 was purely local. In this section we shall discuss how these local solutions may be patched together to form global solutions. The group $G$ may be $S U(2)$ or $S O(3)$. At a general point $p$ of the manifold $M$, the stabilizer of this point must be a discrete subgroup of $G$ since $G$ has 3 dimensional orbits. This subgroup will also fix nearby points since we assume $G$ acts continuously. The 
effective action of $G$ will have this subgroup factored out so we may assume with no loss of generality that $G$ acts effectively and transitively on its orbits $O_{g}$. This implies that these orbits are generically $S^{3}$ if $G=S U(2)$, or $R P^{3}$ if $G=S O(3)$.

In both cases points on $O_{g}$ may be parametrized by the coordinates on $G$ in such a way that the Euler angles $(\psi, \varphi, \vartheta)$ assigned to each point $q$ on the orbit through $p$ correspond to the group element $g$ such that $q=g p$. Since the field equations tell us that the action of $G$ commutes with Lie transport along the orthogonal trajectories this leads to the metric form (4.1) with $\left\{\sigma_{i}\right\}$ given by

$$
\begin{aligned}
& \sigma_{1}=\cos \psi d \vartheta+\sin \psi \sin \vartheta d \varphi \\
& \sigma_{2}=-\sin \psi d \vartheta+\cos \psi \sin \vartheta d \varphi \\
& \sigma_{3}=d \psi+\cos \vartheta d \varphi .
\end{aligned}
$$

The dual basis of vectors $\left\{K_{i}\right\}$ is

$$
\begin{aligned}
& K_{1}=\cos \psi \partial / \partial \vartheta+\frac{\sin \psi}{\sin \vartheta} \partial / \partial \varphi-\cot \vartheta \sin \psi \partial / \partial \psi \\
& K_{2}=-\sin \psi \partial / \partial \vartheta+\frac{\cos \psi}{\sin \vartheta} \partial / \partial \varphi-\cot \vartheta \cos \psi \partial / \partial \psi \\
& K_{3}=\partial / \partial \psi
\end{aligned}
$$

where

$$
\begin{aligned}
& 0 \leqq \vartheta \leqq \pi \\
& 0 \leqq \varphi \leqq 2 \pi \\
& 0 \leqq \psi \leqq 2 \pi \quad \text { if } \quad G \quad \text { is } \quad S O(3) \\
& 0 \leqq \psi \leqq 4 \pi \quad \text { if } \quad G \quad \text { is } \quad S U(2) .
\end{aligned}
$$

The solutions discussed in Sect. 4 are valid so long as $\{a, b, c\}$ are finite and non-zero. If any of $\{a, b, c\}$ cease to be finite and non-zero in a finite proper distance interval $\xi=\int a b c d \eta$, the manifold will be incomplete. If $G$ is $S U(2)$ and all three of $\{a, b, c\}$ diverge as $\frac{1}{2}$ (proper distance) we obviously have a Euclidean infinity. Other infinities are also possible. For example the Taub-NUT infinity corresponds to $a \rightarrow \xi, b \rightarrow \xi, c \rightarrow$ constant (or any permutation of $\{a, b, c\}$ ) as the proper distance $\xi$ tends to infinity. In the Eguchi-Hanson metric $\{a, b, c\}$ diverge as $\frac{1}{2} \xi$ but $G$ is $S O(3)$, giving a sort of "conical" Euclidean infinity [28].

If one of $\{a, b, c\}$ vanishes, for example $c$, at a point $p$, then the corresponding vector will have zero length at $p$. This means that the orbit of $G$ through $p$ can no longer be 3 dimensional. In fact the orbit through $p$ corresponds to a subgroup $H$ of $G$ and hence must be one dimensional or the entire group $G$. In the first case, which we call a bolt, [19] $B$, only $c$ vanishes and the orbit through $p$ corresponds to $G / G_{3}$. This is a homogeneous 2 -space whose metric and second fundamental form are spherically symmetric. In fact since $G$ commutes with the exponential map, $B$ is a totally geodesic submanifold of $M$, and its second fundamental form vanishes. This means that $a$ tends to $b$ on the bolt with vanishing derivative with respect to $\xi$. By considering the limiting form of the metric on a 2 -surface 
orthogonal to the bolt - i.e. the $(\eta, \psi)$ plane in our coordinates - one can readily see that if $\psi$ is to be an angular coordinate with range $4 \pi, c$ must vanish as $\frac{1}{2} \xi$ whereas if $\psi$ has range $2 \pi, c$ must vanish as $\xi$. Strictly speaking the word bolt introduced in [19] applies only when $a=b \forall \xi$. In this case $K_{3}$ is an additional Killing vector (i.e. the group is extended to $U(2))$ and $c=0$ is the locus of its fixed point set - i.e. its "bolt". If $a \neq b$ the set at which $c=0$ is a degenerate orbit but it may not necessarily be the fixed point set of a one parameter subgroup. In what follows we shall extend the term bolt to cover this slightly more general situation.

In the second case the orbit through $p$ is just $p$ itself and we refer to $p$ as a nut [19]. In this case all of $\{a, b, c\}$ must vanish as $\frac{1}{2} \xi$ as $\xi \rightarrow 0$, in order that the orbits be a nested sequence of 3 -spheres near $p$.

We summarize these boundary conditions as follows:

Euclidean Infinity.

$$
\begin{gathered}
a, b, c \rightarrow \frac{1}{2} \xi, \quad \xi \rightarrow \infty \\
0 \leqq \psi \leqq 4 \pi ;
\end{gathered}
$$

Conical Infinity.

$$
\begin{gathered}
a, b, c \rightarrow \frac{1}{2} \xi, \quad \xi \rightarrow \infty \\
0 \leqq \psi \leqq 2 \pi ;
\end{gathered}
$$

Taubian Infinity.

$$
a, b \rightarrow \xi, \quad c \rightarrow \text { constant }, \quad \xi \rightarrow \infty ;
$$

Bolt.

$$
\begin{aligned}
& a, b \rightarrow \text { constant }, \quad c \rightarrow \frac{1}{2} \xi \\
& \frac{d a}{d \xi}, \frac{d b}{d \xi} \rightarrow 0, \quad \xi \rightarrow 0 \\
& 0 \leqq \psi \leqq 4 \pi
\end{aligned}
$$

or

$$
\begin{aligned}
& a, b \rightarrow \text { constant }, \quad c \rightarrow \xi \\
& \frac{d a}{d \xi}, \frac{d b}{d \xi} \rightarrow 0, \quad \xi \rightarrow 0 . \\
& 0 \leqq \psi \leqq 2 \pi ;
\end{aligned}
$$

Nut.

$$
\begin{gathered}
a, b, c \rightarrow \frac{1}{2} \xi, \quad \xi \rightarrow 0 \\
0 \leqq \psi \leqq 4 \pi .
\end{gathered}
$$

There are of course other ways in which the solutions can break down but if we insist on the group orbits being generically $S U(2)$ or $S O(3)$ these solutions will not have regular extensions of the sort we describe. In principle "bolts" with $\psi$ identified modulo $4 \pi / s, s>2$ could arise. These would correspond to group orbits 
which were generically $S U(2) / Z_{s}$ where $Z_{s}$ is the cyclic group of order $s$. However these possibilities definitely do not occur in the symmetric $(a=b)$ case nor the self dual case. If our qualitative reasoning in Sect. 6 is correct they cannot occur at all for Bianchi IX metrics. It will turn out that not all possibilities are allowed by the differential Eqs. (4.4) and (4.5). It is useful to think of the solutions as curves in the configuration space $\{a, b, c\}$.

The five regular boundary conditions can be combined in 15 ways to give regular manifolds. Of these some can be ruled out on the grounds of our results in Sect. 3. These are: Euclidean Infinity $\rightarrow$ bolt or Euclidean Infinity. One can modify our results of Sect. 3 to take into account conical infinities. Each conical infinity, for which $\psi$ is identified modulo $2 \pi$ and the boundary is $R P^{3}$, contributes $\frac{1}{2}$ rather than unity to formula (3.14) for the Euler number. The formula (3.18) for the signature $\tau$ is unchanged. Then it is easy to rule out Conical Infinity $\rightarrow$ Euclidean Infinity, Conical Infinity, or nut. For a Taubian Infinity the boundary terms contribute zero to formula (3.14) for the Euler number and $\frac{1}{3}$ to formula (3.17) for the signature. This enables one to rule out Taubian Infinity $\rightarrow$ Taubian, Euclidean or Conical Infinity.

There are three compact possibilities: nut $\rightarrow$ nut, bolt $\rightarrow$ bolt, and nut $\rightarrow$ bolt. These have $\chi=2,4$ and 3, and $\tau=0,0$ and 1 respectively. They can all be ruled out because

1) If $\chi \neq 0$ any Killing vector field must have at least one fixed point.

2) If $R_{\mu \nu}=0, K_{\mu}^{; \alpha} ; \alpha=0$ which in turn implies that $K_{\mu}$ is covariantly constant and so if zero anywhere it must be zero everywhere.

It remains to investigate the cases

Nut $\rightarrow$ Euclidean Infinity

Nut $\rightarrow$ Taubian Infinity

Bolt $\rightarrow$ Taubian Infinity

Bolt $\rightarrow$ Conical Infinity.

The first case must be flat and the other three all occur as members of the Taub-NUT family - i.e. with two directions equal. One cannot determine by topological means whether the last three cases can occur when all three directions are unequal. To decide the issue we must turn to the detailed behaviour of the differential Eqs. (4.4) and (4.5). This we do in the next section. Before doing so we list the known Taub-NUT examples:

1) Flat Space.

$0 \leqq \psi \leqq 4 \pi$

$a=b=c=\frac{1}{2} \xi$.

This runs from a nut at $\xi=0$ to a Euclidean Infinity at $\xi=\infty$.

2) Self-Dual Taub-NUT [29].

$$
\begin{aligned}
& 0 \leqq \psi \leqq 4 \pi \\
& a=b=\left(r^{2}-n^{2}\right)^{1 / 2}, \quad c=2 n(r-n)^{1 / 2}(r+n)^{-1 / 2} \\
& \xi=n \operatorname{arcosh}\left(\frac{r}{n}\right)+\left(r^{2}-n^{2}\right)^{1 / 2} .
\end{aligned}
$$


This runs from a nut at $\xi=0$ to a Taubian Infinity at $\xi=\infty$.

3) Taub-NUT with $m=\frac{5}{4}|n|$ [31]. (This is not half-flat.)

$$
\begin{aligned}
& 0 \leqq \psi \leqq 4 \pi \\
& a=b=\left(r^{2}-n^{2}\right)^{1 / 2}, \quad c=2 n(r-2 n)^{1 / 2}\left(r-\frac{1}{2} n\right)^{1 / 2}\left(r^{2}-n^{2}\right)^{-1 / 2} \\
& \xi=\int_{2 n}^{r}(x-2 n)^{-1 / 2}\left(x-\frac{1}{2} n\right)^{-1 / 2}\left(x^{2}-n^{2}\right)^{1 / 2} d x .
\end{aligned}
$$

This runs from a bolt at $\xi=0$ to a Taubian Infinity at $\xi=\infty$.

4) Eguchi-Hanson [27, 28].

$$
\begin{aligned}
& 0 \leqq \psi \leqq 2 \pi \\
& a=b=\frac{1}{2} r, \quad c=\frac{1}{2} r\left(1-\frac{a^{4}}{r^{4}}\right)^{1 / 2} \\
& \xi=\int_{a}^{r}\left(x^{4}-a^{4}\right)^{-1 / 2} x^{2} d x .
\end{aligned}
$$

This runs from a bolt at $\xi=0$ to a Conical Infinity at $\xi=\infty$.

Examples (3) and (4) are best understood by comparison with the metric on $C P^{2}$ which has a self-dual Weyl tensor and is an Einstein space [32]. This has

$$
\begin{aligned}
& 0 \leqq \psi \leqq 4 \pi \\
& a=b=\sqrt{\frac{3}{2 \Lambda}} \sin \left(\sqrt{\frac{\Lambda}{6}} \xi\right) \cos \left(\sqrt{\frac{\Lambda}{6}} \xi\right) \\
& c=\sqrt{\frac{3}{2 \Lambda}} \cos \left(\sqrt{\frac{\Lambda}{6}} \xi\right) .
\end{aligned}
$$

This runs from a bolt at $\xi=0$ to a nut at $\xi=\pi \sqrt{\frac{3}{2 \Lambda}}$. Near $\xi=0, c \rightarrow \frac{1}{2} \xi$. The $m=\frac{5}{4}|n|$ Taub-NUT metric has a similar behaviour near the bolt at $\xi=0$. Thus we must identify $\psi$ modulo $4 \pi$. Therefore the topology is that of $C P^{2}-\{$ the point $\left.\xi=\pi \sqrt{\frac{3}{2 \Lambda}}\right\}$. We shall call this $C P^{2}-\{0\}$. It has $\chi=2$ and $\tau=1$. This manifold is simply-connected and does not admit spinor structure because the self-intersection number [19] of the bolt is one, as in $C P^{2}$.

The Eguchi-Hanson metric is also similar to $C P^{2}$ at $\xi \approx 0$, except that $c \rightarrow \xi$ as $\xi \rightarrow 0$. Thus we must identify $\psi$ modulo $2 \pi$, which implies that the Eguchi-Hanson metric has a conical infinity at $\xi=\infty$. In fact the manifold on which the EguchiHanson metric is well-defined may be described as follows. Let $\left(Z_{1}, Z_{2}, Z_{3}\right)$ be three homogeneous complex coordinates on $C P^{2}$, not all of which vanish. Consider the involution $I:\left(Z_{1}, Z_{2}, Z_{3}\right) \rightarrow\left(-Z_{1},-Z_{2},-Z_{3}\right)$. This leaves fixed both the point in $C P^{2}$ given by $\left(0,0, Z_{3}\right)$ which corresponds to the nut, and the complex 
line or 2-sphere $\left(Z_{1}, Z_{2}, 0\right)$ which corresponds to the bolt. If we delete the point and factor $C P^{2}-\{0\}$ by $I$ we obtain the Eguchi-Hanson manifold. (Despite appearances this is still a manifold near the bolt, and is in fact diffeomorphic to the tangent bundle of $S^{2}$.)

Introducing coordinates

$$
\begin{aligned}
& Z_{1} / Z_{3}=\zeta^{1}=R \cos \left(\frac{1}{2} \vartheta\right) \exp \left(\frac{i}{2}(\psi+\varphi)\right) \\
& Z_{2} / Z_{3}=\zeta^{2}=R \sin \left(\frac{1}{2} \vartheta\right) \exp \left(\frac{i}{2}(\psi-\varphi)\right)
\end{aligned}
$$

where $R^{2}=\left|\zeta^{1}\right|^{2}+\left|\zeta^{2}\right|^{2}$, it is evident that the involution $I$ corresponds to $\psi \rightarrow \psi+2 \pi$.

$\left(C P^{2}-\{0\}\right) / I$ has $\chi=2, \tau=1$ (the same as $\left.C P^{2}-\{0\}\right)$ and is simply-connected. The self-intersection number [19] of the bolt is 2 and the manifold admits spinor structure. One may easily check the index theorems for the Hirzebruch and Dirac complexes. The $R P^{3}$ boundary contributes zero for the Hirzebruch case and $-\frac{1}{8}$ for the Dirac case.

The remaining explicitly known solution, that of Belinskii, Gibbons, Page and Pope [28], Eq. (4.23), in general runs from a Euclidean Infinity at $\varrho=\infty$ to a curvature singularity at the largest value of $\left(a_{1}, a_{2}, a_{3}\right)$, except in the special case where it coincides with the Eguchi-Hanson metric. In any event it is not complete and asymptotically Euclidean. Since it is half-flat this is implied by our results in Sect. 3 .

\section{Qualitative Treatment of Bianchi IX Solutions}

The Eqs. (4.4) and (4.5) are difficult to integrate exactly. Similar equations (obtained by Wick rotation) occur in the study of cosmological models, and various qualitative methods have been developed to deal with them. In this section we shall adapt the approach of Misner [33] to our situation. We begin by introducing the variables $\left(\Omega, \beta_{+}, \beta_{-}\right)$defined by

$$
\begin{aligned}
& a=\exp \left(\Omega+\beta_{+}+\sqrt{3} \beta_{-}\right) \\
& b=\exp \left(\Omega+\beta_{+}-\sqrt{3} \beta_{-}\right) \\
& c=\exp \left(\Omega-2 \beta_{+}\right)
\end{aligned}
$$

or

$$
\begin{aligned}
& \Omega=\frac{1}{3} \log (a b c) \\
& \beta_{+}=\frac{1}{6} \log \left(\frac{a b}{c^{2}}\right) \\
& \beta_{-}=\frac{1}{2 \sqrt{3}} \log \left(\frac{a}{b}\right) .
\end{aligned}
$$


The action $I$ in (4.7) is now

$$
I=\frac{3 \pi}{4} \int\left[\left(\frac{d \beta_{+}}{d \eta}\right)^{2}+\left(\frac{d \beta_{-}}{d \eta}\right)^{2}-\left(\frac{d \Omega}{d \eta}\right)^{2}-\Phi e^{4 \Omega}\right] d \eta
$$

where

$$
\begin{aligned}
\Phi\left(\beta_{+}, \beta_{-}\right)= & -\frac{1}{12}\left[2 e^{4 \beta_{+}} \cosh \left(4 \sqrt{3} \beta_{-}\right)-2 e^{4 \beta_{+}}\right. \\
& \left.-4 e^{-2 \beta_{+}} \cosh \left(2 \sqrt{3} \beta_{-}\right)+e^{-8 \beta_{+}}\right] .
\end{aligned}
$$

The constraint Eq. (4.6) becomes

$$
\left(\frac{d \beta_{+}}{d \eta}\right)^{2}+\left(\frac{d \beta_{-}}{d \eta}\right)^{2}-\left(\frac{d \Omega}{d \eta}\right)^{2}+\Phi e^{4 \Omega}=0 .
$$

These equations can be thought of as those of a particle moving in the timedependent potential $\Phi e^{4 \Omega}$. For some purposes it is convenient to regard $\Omega$ as the time coordinate. At large negative values of $\Omega$ the potential is flat. As $\Omega$ increases so does the potential, becoming infinitely steep as $\Omega \rightarrow \infty$. Care must be taken however since $\Omega$ is not always a monotonic function of $\eta$ or $\xi$.

The self-dual first integrals may be written in the form

$$
\begin{gathered}
\frac{d \Omega}{d \eta}=\partial / \partial \Omega\left(e^{2 \Omega} \Psi_{i}\left(\beta_{+}, \beta_{-}\right)\right) \\
\frac{d \beta_{+}}{d \eta}=-\partial / \partial \beta_{+}\left(e^{2 \Omega} \Psi_{i}\left(\beta_{+}, \beta_{-}\right)\right) \\
\frac{d \beta_{-}}{d \eta}=-\partial / \partial \beta_{-}\left(e^{2 \Omega} \Psi_{i}\left(\beta_{+}, \beta_{-}\right)\right)
\end{gathered}
$$

where for case $C$ (the $B G P^{2}$ solutions) $i=1$ and

$$
\Psi_{1}=\frac{1}{12}\left(2 e^{2 \beta_{+}} \cosh \left(2 \sqrt{3} \beta_{-}\right)+e^{-4 \beta_{+}}\right)
$$

and for case $D$ (which we cannot integrate exactly) $i=2$ and

$$
\Psi_{2}=-\Phi\left(\frac{\beta_{+}}{2}, \frac{\beta_{-}}{2}\right) \text {. }
$$

Both $\Psi_{1}$ and $\Psi_{2}$ are invariant under rotations of the $\left(\beta_{+}, \beta_{-}\right)$plane through $\pm \frac{2}{3} \pi$, and reflections in the $\beta_{+}$axis. This is the action of the permutation group on 3 objects and corresponds to interchanging the roles of $(a, b, c)$.

The potential $\Phi\left(\beta_{+}, \beta_{-}\right)$has the following further properties. Near the origin it has the form

$$
\Phi=\frac{1}{4}-2\left(\beta_{+}^{2}+\beta_{-}^{2}\right)+O\left(\beta^{3}\right) .
$$

$(0,0)$ is a local maximum. For large negative $\beta_{+}$such that $2\left|\beta_{-}\right|<\sqrt{3}\left|\beta_{+}\right|$it falls rapidly to $-\infty$ and

$$
\Phi \approx-\frac{1}{12} e^{8 \mid \beta+1} .
$$

For large positive $\beta_{+}$and small $\beta_{-} \neq 0$,

$$
\Phi \approx-4 \beta_{-}^{2} e^{4 \beta_{+}}
$$




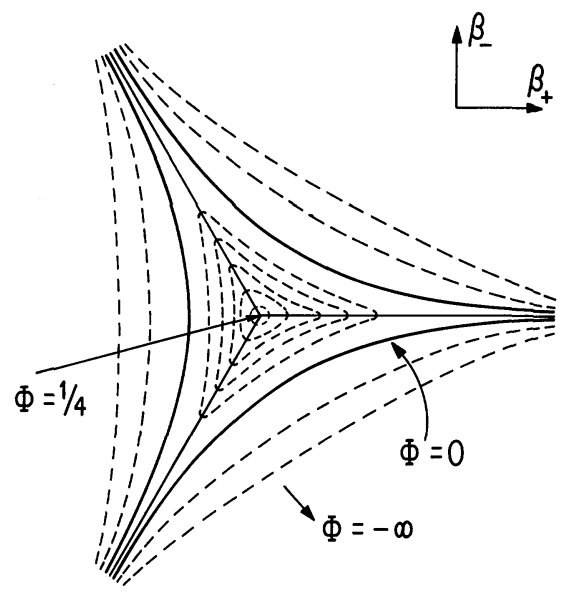

Fig. 1. A sketch of the potential $\Phi\left(\beta_{+}, \beta_{-}\right)$. The level curves are shown dotted. The potential may be envisaged as 3 gently sloping ridges which meet at the highest point of the potential in the centre. To either side of the ridges the potential falls rapidly down three steep cliffs to $-\infty$. $\Phi$ is positive near the centre and passes through zero on the level curve indicated with a solid line

while for $\beta_{-}=0$,

$$
\Phi \approx \frac{1}{3} e^{-2 \mid \beta+1} .
$$

The equipotential curves are circular near the origin and become more triangular as one moves outward, until at the equipotential $\Phi=0$ they become three disjoint open curves, asymptotic to the positive $\beta_{+}$and symmetry-related axes. Outside $\Phi=0$ they become straighter and straighter. Thus the potential landscape may be envisaged as three vertical cliffs meeting at three ridges which slope downwards rather gently (see Fig. 1). This potential is the negative of the usual one encountered in Bianchi IX cosmological models because we are working in imaginary time.

The various boundary conditions and specializations we have described previously may be translated in terms of this simple model. Clearly the trajectories for which $a=b$ are those which have $\beta_{-}=0$ and move straight down the cliffs or along the ridges. The volume of the group orbits is $16 \pi^{2} e^{3 \Omega}$ or $8 \pi^{2} e^{3 \Omega}$ depending upon whether the orbits are $S^{3}$ or $R P^{3}$. Thus an asymptotically flat region corresponds to large positive values of $\Omega$ and a nut or bolt to large negative values. One can easily see that the various possibilities are given by

Euclidean or Conical Infinity.

$$
\begin{aligned}
\Omega \rightarrow \infty, & \frac{d \Omega}{d \eta} \rightarrow 0, \\
\left(\beta_{+}, \beta_{-}\right) \rightarrow 0, & \left(\frac{d \beta_{+}}{d \eta}, \frac{d \beta_{-}}{d \eta}\right) \rightarrow 0, \\
\eta \rightarrow \eta_{0} & \text { (finite). }
\end{aligned}
$$


Taubian Infinity.

$$
\begin{gathered}
\Omega \rightarrow \infty, \quad \frac{d \Omega}{d \eta} \rightarrow \infty, \\
\beta_{+} \rightarrow \infty, \quad \frac{d \beta_{+}}{d \eta} \rightarrow \infty, \\
\beta_{-} \rightarrow 0, \quad \frac{d \beta_{-}}{d \eta} \rightarrow 0, \\
\eta \rightarrow \eta_{0} \quad \text { (finite). }
\end{gathered}
$$

Bolt.

$$
\begin{aligned}
& \Omega \rightarrow-\infty, \quad \frac{d \Omega}{d \eta} \rightarrow \frac{n a_{0}^{2}}{6}, \\
& \beta_{+} \rightarrow \infty, \quad \frac{d \beta_{+}}{d \eta} \rightarrow-\frac{n a_{0}^{2}}{6}, \\
& \beta_{-} \rightarrow 0, \quad \frac{d \beta_{-}}{d \eta} \rightarrow 0,
\end{aligned}
$$

where

$$
\begin{aligned}
& n=1 \text { for } S^{3} \\
& n=2 \text { for } R P^{3} .
\end{aligned}
$$

Nut.

$$
\begin{gathered}
\Omega \rightarrow-\infty, \quad \frac{d \Omega}{d \eta} \rightarrow 0, \\
\left(\beta_{+}, \beta_{-}\right) \rightarrow 0, \quad\left(\frac{d \beta_{+}}{d \eta}, \frac{d \beta_{-}}{d \eta}\right) \rightarrow 0, \\
\eta \rightarrow \infty
\end{gathered}
$$

The trajectory of the fictitious particle moving in the potential $\Phi e^{4 \Omega}$ can begin or end at one of three points (modulo permutations of $(a, b, c))$ in the $\left(\beta_{+}, \beta_{-}\right)$ plane. They are the Summit $\left(\beta_{+}=\beta_{-}=0\right)$, the End of the Ridge $\left(\beta_{+}=\infty, \beta_{-}=0\right)$, and the Bottom of the Cliff $\left(\beta_{+}=-\infty, 2\left|\beta_{-}\right|<\sqrt{3}\left|\beta_{+}\right|\right)$. These correspond to the possible ways in which $(a, b, c)$ can cease to be finite and non-zero. Provided the velocity of the particle is also appropriate, the Summit corresponds to a nut if $\Omega=-\infty$, and a Euclidean or Conical Infinity if $\Omega=+\infty$. Similarly the End of the Ridge corresponds to a bolt if $\Omega=-\infty$ and a Taubian Infinity if $\Omega=+\infty$. The Bottom of the Cliff is singular and the curvature diverges there.

The self-dual class $C$ metrics correspond to the set of all trajectories which just reach the origin as $\Omega \rightarrow+\infty$. The regular Eguchi-Hanson metric (type II) corresponds to such a trajectory that starts from the End of the Ridge at $\Omega=-\infty$. The incomplete Eguchi-Hanson metric (type I) corresponds to a trajectory which starts from the Bottom the Cliff on the $\beta_{+}$axis at $\Omega=-\infty$. The general class $C$ 
metrics (i.e. the $B G P^{2}$ solutions, Eq. (4.23)) correspond to the remaining "generic" trajectories - i.e. those which start at the Bottom of the Cliff at $\Omega=-\infty$ and for which $a, b$ and $c$ are all unequal.

The self-dual Taub-NUT solution (Eq. (4.10) with $m= \pm|n|$ ) corresponds to trajectories which start with zero velocity at the Summit at $\Omega=-\infty$ and fall along the $\beta_{+}$axis reaching either the End of the Ridge (if $m=+|n|$ ) or the Bottom of the Cliff (if $m=-|n|$ ) as $\Omega \rightarrow+\infty$. The former corresponds to the complete nonsingular manifold for which $0<m \leqq r<\infty$. The general two parameter Taub-NUT solution (Eq. (4.10)) is represented by the set of all trajectories along the $\beta_{+}$axis which start at $\Omega=-\infty$ from either the End of the Ridge or the Bottom of the Cliff and which do not arrive at the Summit with zero velocity at $\Omega=+\infty$. That is they have insufficient energy to reach the Summit and so fall back again, or else they overshoot. The first of these possibilities corresponds to the usual outer region of Taub-NUT space $\left(m+\left(m^{2}-n^{2}\right)^{1 / 2}<r<\infty\right)$. In the special case $m=\frac{5}{4}|n|$ the particle sets out as a bolt at $\Omega=-\infty$, travels some way up the ridge and falls down again to arrive at the end as a Taubian Infinity at $\Omega=+\infty$.

The general self-dual class $D$ metrics (Eqs. (6.10), (6.11), (6.12) with $i=2$ ) can be studied qualitatively in the same way. The trajectories in the $\left(\beta_{+}, \beta_{-}\right)$plane are lines of steepest ascent or descent of the potential $e^{2 \Omega} \Phi\left(\beta_{+} / 2, \beta_{-} / 2\right)$. This follows directly from Eqs. (6.11) and (6.12). Therefore unless the trajectories start out along one of the ridges they will inevitably end up at the Bottom of the Cliff. Thus the self-dual Taub-NUT metric is the only complete non-singular solution in this class. In fact it follows directly from our results in Sect. 3 that any self-dual trajectory which end on the Euclidean Infinity must have started from the bottom of a cliff.

The most general Bianchi IX case (Eqs. (4.4), (4.5)) is rather complicated. It is clear that solutions cannot leave the Summit and return later. This therefore rules out non-flat Bianchi IX metrics on $R^{4}$ (nut $\rightarrow$ Infinity), $S^{3} \times R$ (Infinity $\rightarrow$ Infinity) or $S^{4}$ (nut $\rightarrow$ nut). These are ruled out anyway by our results in Sects. 3 and 5 . It also seems clear that a trajectory which leaves the Summit not directed along a ridge cannot subsequently return to a ridge to become a bolt or Taubian Infinity.

It is not obvious to us whether trajectories can start out from the end of one ridge as a bolt or Taubian Infinity and finish up at the end of another ridge as a Taubian Infinity or bolt respectively, but we regard this as unlikely. The other two possibilities (bolt $\rightarrow$ bolt or Taubian Infinity $\rightarrow$ Taubian Infinity) are ruled out by our results in Sects. 5 . Thus it seems rather likely that the only regular Bianchi IX solutions have the additional symmetry that two of the directions are equal.

\section{Conclusion}

An asymptotically Euclidean metric with $N$ asymptotic regions is a complete nonsingular metric on a manifold region diffeomorphic to the union of a compact set $K$ and $N$ complements of the open ball in $R^{4}$ on each of which the metric has the form

$$
g_{\mu \nu}=\left(1+\frac{\tilde{I}}{6 \pi r^{2}}\right)^{2} \delta_{\mu \nu}+O\left(\frac{1}{r^{3}}\right)
$$


If $R_{\sigma}^{\sigma}=0$ the action of this region is $I=\tilde{I}$. The Strong Positive Action Conjecture is that $R_{\sigma}^{\sigma}=0$ implies $I \geqq 0$, with $I=0$ iff $g_{\mu v}$ is flat. This implies the Weak Positive Action Conjecture i.e. $R_{\mu \nu}=0$ implies $g_{\mu \nu}$ is flat.

In this paper we prove

1) There are no half flat asymptotically Euclidean metrics with finite fundamental group other than flat space.

2) Any non-flat asymptotically Euclidean solution with Euler number $\chi$ and signature $\tau$ must satisfy $2 \chi-3|\tau|>2 N$. In particular there are no non-flat solutions on $R^{4}$.

We have illustrated these results by studying a class of Bianchi IX solutions with $S U(2)$ or $S O(3)$ as isometry group. We have shown (modulo one possible but unlikely case) that the only instantons of this class must possess a further symmetry and we have given the explicit solutions. In an appendix we use these results to give an approximate construction for the only self-dual compact instanton.

Asymptotically Euclidean instantons, if they existed, would contribute to the vacuum-vacuum amplitude for gravity. Instantons with more than one asymptotically Euclidean region may lead to baryon non-conservation. The fact that any such configuration is (by the Cosmic Censorship Hypothesis) far from an extremum of the gravitational action presumably means that such processes are forbidden in the semi-classical approximation.

Another mechanism, due to 't Hooft, for baryon non-conservation requires the existence of bound states of the Dirac equation. These cannot occur on noncompact gravitational backgrounds with $R_{\mu}^{\mu} \geqq 0$.

These two facts tend to suggest that for pure gravity coupled to ordinary matter the baryon non-conserving processes are forbidden to lowest order in the stationary phase approximation. This situation changes if one includes spin $3 / 2$ fields as part of a supersymmetric theory of gravity.

\section{Appendix. An Approximate Metric on $K 3$}

In this appendix we shall describe how 16 Eguchi-Hanson spaces may be "glued" together to give a self-dual metric on a $K 3$ surface. Hitchin [20] has shown that all compact half-flat spaces are homeomorphic to a $K 3$ surface or an identification there of. All $K 3$ surfaces are diffeomorphic to any quartic surface in $C P^{3}$. A particular such surface is a Kummer Surface. This is holomorphically equivalent to the following space [34]: Consider a lattice $L$ in $C^{2}$ obtained by identifying the points $\left(Z_{1}, Z_{2}\right)$ with $\left(Z_{1}, Z_{2}\right)+\lambda(1,0)+\mu(i, 0)+v(0,1)+\sigma(0, i)$, where $(\lambda, \mu, v, \sigma)$ are 4 arbitrary integers. This is a compact complex manifold with topology $S^{1} \times S^{1} \times S^{1} \times S^{1}$. The involution $\left(Z_{1}, Z_{2}\right) \rightarrow\left(-Z_{1},-Z_{2}\right)$ has 16 fixed points in $L$, i.e. the points whose real and imaginary parts are zero or $1 / 2$. If one identifies points in $L$ which are equivalent under the involution these 16 points will not be regular points. One must, in the language of algebraic geometers, "blow them up". Roughly, this means replacing these 16 points by 16 copies of $C P^{1}$ - the complex projective line or Riemann sphere. This may be done as follows. Surround each of the 16 points by small 3 -spheres and remove the interiors. The boundary of the manifold so obtained is 16 disjoint copies of $R P^{3}$. It is $R P^{3}$ and not $S^{3}$ because the 
involution connects antipodal points on $S^{3}$. Each $R P^{3}$ boundary must now be filled in with an Eguchi-Hanson manifold (i.e. $\left.\left(C P^{2}-\{0\}\right) / I\right)$. To construct the metric one has to match carefully across these 16 boundaries. This can be done approximately by putting the flat metric on $C^{2}$ and the Eguchi-Hanson metric on the Eguchi-Hanson manifold. As their parameter $a \rightarrow 0$ this will become a better and better fit. It is easy to check that the Euler number $\chi$ and signature $\tau$ of the resulting space are 24 and 16 respectively and that this is consistent with the Gauss-Bonnet and signature theorems. Page [35] has established that the number of free parameters or moduli obtained from this construction corresponds exactly with the 58 expected ones. If one could find a "multi-Eguchi-Hanson" solution analogous to the multi Taub-NUT solution [29] one might hope that by judicious choice of the parameters one could construct exactly the general self-dual metric on $K 3$.

In fact a minor modification of the multi-taub-NUT ansatz produces a sequence of solutions which are asymptotically conical [19]. The first of the sequence in flat space which is of course asymptotically Euclidean and the second is Eguchi-Hanson with boundary $R P^{3}$. As one proceeds along the sequence the metrics are all asymptotically flat in all 4 directions but with lens space boundaries. However each of the solutions has at least a one parameter isometry group and so superposing will not lead directly to the desired metric on $K 3$. Further work on this topic is continuing.

Acknowledgements. We would like to thank J. F. Adams, M. F. Atiyah, S. W. Hawking, N. J. Hitchin, P. J. McCarthy, and D. N. Page for conversations and help.

\section{References}

1. Harrison, B.K., Thorne, K.S., Wakane, M., Wheeler, J.A.: Gravitation theory and gravitational collapse, pp. 9 and 146. Chicago, London: University of Chicago Press 1965

2. Hawking, S.W.: Commun. math. Phys. 43, 199-220 (1975)

3. Zeldovich, Ya.B.: Phys. Lett. 59 A, 254 (1976); 73B, 423-424 (1978)

4. Gibbons, G.W., Hawking, S.W.: Phys. Rev. D15, 2752-2756 (1977)

5. Gibbons, G.W., Hawking, S.W., Perry, M.J.: Nucl. Phys. B 138, 141-150 (1978)

6. Page, D.N.: The positive action conjecture. Phys. Rev. D 18, 2733-2738 (1978)

7. Schoen, R., Yau, S.T.: Proc. Natl. Acad. Sci. U.S. 75, 2567 (1978); Schoen, R., Yau, S.T.: Commun. Math. Phys. 65, 45-76 (1979)

8. 't Hooft, G.: Phys. Rev. Lett. 37, 8-11 (1976); Phys. Rev. D 14, 2432-2450 (1976)

9. Hawking, S.W.: Phys. Rev. D 18, 1747-1753 (1978), and various unpublished lectures

10. Lichnerowicz, A.: Compte Rendue 257, 5-9 (1968)

11. Hawking, S.W., Pope, C.N.: Nucl. Phys. B 146, 381-392 (1978)

12. Misner, C.W., Wheeler, J.A.: Ann. Phys. N.Y. 2, 525-660 (1957)

13. Misner, C.W.: Phys. Rev. 118, 1110 (1960)

14. Misner, C.W.: Ann. Phys. N.Y. 24, 102-117 (1963)

15. Gibbons, G.W.: Commun. Math. Phys. 27, 87-103 (1972)

16. Gibbons, G.W., Schutz, B.F.: M.N.R.A.S. 159, 41-45 (1972)

17. Penrose, R.: Ann. Acad. Sci. 224, 125 (1973)

18. Jang, P.S., Wald, R.M.: J. Math. Phys. 18, 41-44 (1977)

19. Gibbons, G.W., Hawking, S.W.: Classification of gravitational instanton symmetries. Commun. Math. Phys. (in press) 
20. Hitchin, N.: J. Diff. Geom. 9, 435-441 (1975)

21. Tolman, R.C.: Relativity, thermodynamics and cosmology. London: O.U.P. 1934

22. Chern, S.: Ann. Math. 46, 674 (1945)

23. Eguchi, T., Gilkey, P., Hanson, A. : Phys. Rev. D 17, 423-427 (1978)

24. Atiyah, M.F., Patodi, V.K., Singer, I.M. : Math. Proc. Cambridge Phil. Soc. 77, $43-69$ (1975)

25. Hawking, S.W., Pope, C.N.: Phys. Lett. 73B, 42 (1978)

26. Landau, L., Lifshitz, E.M.: Classical theory of fields. 4th Ed. Oxford, New York : Pergamon 1975

27. Eguchi, T., Hanson, A.: Phys. Lett. 74B, 249-251 (1978)

28. Belinskii, V.A., Gibbons, G.W., Page, D.N., Pope, C.N.: Phys. Lett. 76B, $433-435$ (1978)

29. Hawking, S.W.: Phys. Lett. 60 A, 81-82 (1977)

30. Atiyah, M.F., Hitchin, N., Singer, I.M.: Proc. R. Soc. A362, 425 (1978)

31. Page, D.N.: Phys. Lett. 78B, 249-251 (1978)

32. Gibbons, G.W., Pope, C.N.: Commun. Math. Phys. 61, 239-248 (1978)

33. Misner, C.W., Thorne, K.S., Wheeler, J.A.: Gravitation. San Francisco: Freeman 1970

34. Lascoux, A., Berger, M.: Varietes Kahleriennes compactes. In: Lecture notes in mathematics, Vol. 154. Berlin, Heidelberg, New York: Springer 1970

35. Page, D.N. : Phys. Lett. 80B, 55-57 (1978)

Communicated by R. Geroch

Received October 13, 1978 